\title{
Assessment of Quality of Speech Transmitted over IP Networks
}

\author{
Stefan Brachmański \\ Institute of Telecommunications and Acoustics, Wroclaw University of Technology, Wroclaw, \\ Poland \\ brach@aipsa.ita.pwr.wroc.pl
}

\begin{abstract}
This paper presents subjective methods of speech transmission quality evaluation in packets (IP) networks. It includes a review of selected methods of subjective listening measurements recommended by ITU-T, the Polish Standard and new test called "modified intelligibility test with forced choice" (MIT-FC).
\end{abstract}

Key words: speech quality, speech intelligibility, VoIP

\section{INTRODUCTION}

Telephone is the most widespread mean of message transmission. In a conventional telephone network, known as the Public Switched Telephone Network (PSTN), speech is transmitted in an analog or digital line. In recent years the integration of voice with data, in a common packet switched network such as Asynchronous Transfer Mode (ATM), Frame Relay (FR) and Internet Protocol (IP) is observed. The integration of voice with data offers potential cost savings, because transmission of packets takes place only when speakers are speaking.

Voice over Internet Protocol (VoIP) is the most popular technique of speech transmission in packet networks. This technology uses data networks such as the Internet to transmit voice information by means of a simple PC via microphone and loudspeaker connected to the sound card. VoIP is primarily a software feature.

VoIP technology, as with all telecommunication technology, is governed by sets of standards that have been established by the International 
Telecommunication Union (ITU). Now it appears that most have accepted the ITU's H.323 global standard for packet-based multimedia communication like VoIP. Speech communication requires networks with very low latency (delay), low jitter (variable delays) and minimal packet loss (dropped packets). Low latency allows users to carry on natural conversations. The human ear can tolerate some latency, usually up to around $250 \mathrm{~ms}$. Hovewer, most VoIP sessions require one-way latency of not more than about $150 \mathrm{~ms}$, at a packet loss of less than $5 \%$. When delay excceed approximately $300 \mathrm{~ms}$, natural conversation becomes difficult. A delay of $400 \mathrm{~ms}$ is not acceptable for professional use. However, for private use a delay exceeding up $400 \mathrm{~ms}$ can be tolerated even though the connection is not satisfactory [13]. Monitoring the total delay is necessary in maintaining a good quality VoIP transmission.

VoIP introduces many problems with speech quality. Speech quality is very important and can vary onVoIP networks. The gateway equipment, the phone systems, software and carrier infrastructure have an effect on speech quality.

For the data transmission the low quality of IP global network has a minor importance, because the packets received in wrong order may be ranked, delayed ones can be awaited and lost or damaged packets can be sent again. On the contrary, the speech transmission demands adequate quality of the network, because too big delays or lost packets can influence the legibility of speech.

For the methods of quality evaluation of services (including speech transmission in joined IP/PSTN networks) fulfilling the rules of universality (measure of "end-to-end" class independently of transmission and coding type), repetition and instrumental-objectivity is demanded. In current research works and normalization arrangements concerning methods of quality evaluation of transmission, given above conditions implied concentration on a new generation of objective methods which are effective for various types of telephone channels (including those with nonlinear distortions and changeable in time delays "bulk delays") and simulating in maximum way subjective measurement.

Despite the crucial progress of instrumental measurement techniques of this parameter, the only reliable method is still a very time-consuming, expensive and high skill-demanding subjective measurement of intelligibility done with group of specially trained listeners.. Designers of speech transmission quality measurement systems try to apply objective measurement techniques not always regarding the limitations and accuracy of measure. Yet the final verification of quality of devices used for speech transmission is still done by their user-human. 
Among various subjective techniques, often from the beginning of $\mathrm{XX}$ century, nowadays are in use techniques, which give the Mean Opinion Score (MOS) in five levels scale directly (ACR) or indirectly (measurement of intelligibility, DRT method-Diagnostic Rhyme Test and MRT-Modified Rhyme Test) $[4,5,7,9,11,12]$. Despite the way of obtaining the MOS, the integral part of the method should be the measure of reference letting for comparison and averaging MOS for various methods, conditions and languages. For digital chanels the MNRU method is used according to ITU recommendations [6].

\section{LISTENING TEST - ABSOLUTE CATEGORY RATING (ACR)}

The method ACR is recommended by International Telecommunication Union (ITU) to evaluation of speech transmission quality in analog and digital telecommunications channels and speech coding systems [5].

The speech material used in this method should consist of simple, meaningful, short sentences. These sentences should be set into phoneticaly balanced lists. Each list should consist of five or ten groups. The experimenter must decide how many sentences (a minimum of two and a maximum five sentences) are required in each group to constitute a speech sample. The test material should be suitably prepared and registered. Speaker should speak the sentences clearly, fluently with equal loudness, and have no speech deficiencies such as stutter, lisp, etc. To reduce influence of individuality of the voices on result of assessment, the measurement should be carryied out for more than one male and more then one female voice.

The speaker should be seated in a quiet room with volume between 30 and $120 \mathrm{~m}^{3}$ and a reverberation time less than $0.5 \mathrm{~s}$ (preferably in the range $0.2-0.3 \mathrm{~s}$ ). The room noise level must be below $30 \mathrm{dBA}$ with no dominant peaks in the spectrum.

The test material spoken by speaker can be recorded on the high quality recording system of the following:

- a conventional two-track tape recorder,

- a Digital Audio Tape recorder,

- a computer with acoustic input and output.

The active speech level during the recording process should be set up to the range of $20-30 \mathrm{~dB}$ below the overload point of the recording system. At the beginning of each recording there are 20 seconds calibration tone of known level. This calibration tone is normally at $1000 \mathrm{~Hz}$. If the recordings are intended for playing through systems that respond to $1000 \mathrm{~Hz}$, the tone 
may be of some other frequency. This calibration tone can be used to adjust the listening levels.

Every experiment should include reference conditions so that experiments made in different laboratories or at a different time in the same laboratory can be sensibly compared. For telecommunication systems the reference conditions may include the Modulated Noise Reference Unit [6].

The listeners are seated in a quiet room with volume between 30 and $120 \mathrm{~m}^{3}$ and a reverberation time less than $0.5 \mathrm{~s}$ (preferably in the range $0.2-0.3 \mathrm{~s}$ ). The room noise level must be below $30 \mathrm{dBA}$ with no dominant peaks in the spectrum. It is recommended that the noise level and spectrum are measured at least twice; at the beginning and end of the measurements. If there is any difference between the two measurements, the operator must be assessed as it may cast doubt the result of the experiment.

Listeners are chosen at random from the normal telephone using population, with the provisions that:

- they have not been involved in work connected with assessment of performance of telephone systems or speech coding,

- they have not participated in any subjective measurements at least the previous six months,

- they have never heard the same sentences lists before.

Listeners read instruction of experiment before beginning measurements. Various scales may be used for different purposes. Operator give the following opinion scales recommended by ITU [5]:

a) listening-quality scale (Excellent speech is rated 5, Good - 4, Fair - 3, Poor - 2, Bad - 1),

b) listening-effort scale(Complete relaxation possible; no effort required is rated 5, Attention necessary; no appreciable effort required - 4, Moderate effort required - 3, Considerable effort required - 2, No meaning understood with any feasible effort - 1),

c) loudness-preference scale (Much louder than preferred is rated 5, Louder than preferred - 4, Preferred - 3, Quieter than preferred - 2, Much quieter than preferred - 1).

The mean value should be calculated over listeners and speakers for each condition of speech transmission. Averaging the scores of the male and femal talkers should be made with care.

\section{SUBJECTIVE MEASUREMENTS OF LOGATOM INTELLIGIBILITY - TRADITIONAL METHOD}

The traditional measurement method of speech intelligibility is based on transmission of logatom test lists via telecommunication channel and the 
listeners who hav e to write what they hear [11]. In the next step, the group of experts checks the correctness of received logatoms and the average logatom intelligibility is calculated in accordance to Eq. (1) and (2). Lists consisted of 50 or 100 logatoms (i.e. nonsense pseudo-words) are recommended and each list has to be phonetically and structurally balanced.

The logatoms should be spoken with equal loudness, clearly without stresses at the beginning of logatoms or on vowels. The time interval between logatoms should allow the listeners to write received logatoms ( 3 to 5 second is recommended). The interval between session should be no shorter than $24 \mathrm{~h}$ and no longer than 3 days. The whole session time should not exceed $3 \mathrm{~h}$ (together with $10 \mathrm{~min}$ breaks after every 20 min of listening).

Listeners write received logatoms on special sheets and the day, test list number, name or symbol of speaker, name of listener and other specific information are noticed. The test sheets should be written clearly to avoid errors in logatom interpretation. Received logatoms may be written in phonetic transcription (the listeners must know it) or in traditional way, specific for a given language. Experts, who check the lists, calculate the number of correct responses for each listener and each logatom list, and then average logatom intelligibility is determined.

The average logatom intelligibility $W_{L}$ is calculated from the relationship:

$$
W_{L}=\frac{1}{N K} \sum_{n=1}^{N} \sum_{k-1}^{K} W_{n, k} \quad[\%]
$$

where: $N$ - number of listeners,

$K$ - number of test lists,

$W_{n, k}$ - logatom intelligibility for $n$-listener and $k$-logatom list,

$$
W_{n, k}=\frac{P_{n, k}}{T_{k}} \cdot 100 \quad[\%]
$$

where: $P_{n, k}$ - number of correctly received logatoms from $k$-test list by $n$-listener, $T_{k}$ - number of logatoms in $k$-test list.

Standard deviation $s$, calculated in accordance to Eq.(3), expresses the distribution of logatom intelligibility values $W_{L}$ over listeners.

$$
s=\left[\frac{1}{N \cdot K-1} \sum_{n=1}^{N} \sum_{k=1}^{K}\left(W_{n, k}-W_{L}\right)^{2}\right]^{1 / 2}
$$

If $\left|W_{n, k}-W_{L}\right|>3 s$, the result of measurement is not taken into account, when the average intelligibility is calculated and calculation of $W_{L}$ and $s$ must be done in accordance to Eq. (1) and (2) for reduced number of measurements. 


\section{MODIFIED INTELLIGIBILITY TEST WITH FORCED CHOICE (MIT-FC)}

The subjective measurement of logatom intelligibility is very time- and cost-consuming, because it is necessary to have a high-specialized group of experts as the listener staff in order to obtain the high reliability of measurement results. To avoid disadvantages of subjective evaluation of logatom intelligibility by means of "traditional" method, a new measurement method was created and developed at the Institute of Telecommunications and Acoustics. This method is called "modified intelligibility test with forced choice" (MIT-FC) [1, 9]

In the MIT-FC method a computer controls all experiments. The automation of the subjective measurement is connected with the basic change in generation of logatoms and in making decision by a listener. The computer generates a list of utterences (for logatom test the list consists of 100 phonetically balanced nonsense words), presents the utterances to the listeners subsequently and for each spoken utterance there are visually presented several logatoms that have been previously selected as perceptually similar. It has been found $[1,2]$ that the optimal number of logatoms presented visually to the listeners is seven (six alternative logatoms and one transmitted logatom to be recognized). The listener chooses one logatom from the list visually presented on the computer monitor. The computer counts the correct answers and calculates the average logatom intelligibility and standard deviation. The measurement time for one logatom list consisted of 100 logatoms is $8-9$ minutes. All measurement procedures are fully automatized and an operator has a flexible possibilities to set the measurement parameters and options. It is also possible to upgrade the application that realizes the MIT-FC method with more sophisticated scores processing.

The MIT-FC method was implemented in a stationary version for the measurements of telecommunication systems and chains and in a portable version for the measurements of auditory and mobile communication systems. The stationary version was implemented on the PC with 16 bit A/D and D/A conversion and the portable version was implemented on the Laptop Toshiba TECRA CDT 720 with similar sound card.

Block diagram system for the subjective measurements of logatom intelligibility by MIT-FC method in the IP networks is presented on Figure 1. All the measurement processes are controlled by computer program. This program takes the samples of test signal (logatom) from a computer memory and sends the logatom into investigated telecommunication channel via $\mathrm{D} / \mathrm{A}$ converter. Next, the signal is amplified and given to the headphones. Each listener has a computer monitor and a keybord. The listener listenes and 
chooses one logatom from the list visually presented on the computer monitor. The number of alternative logatoms is set-up by operator before beginning of an experiment (optimal number is seven [1]).

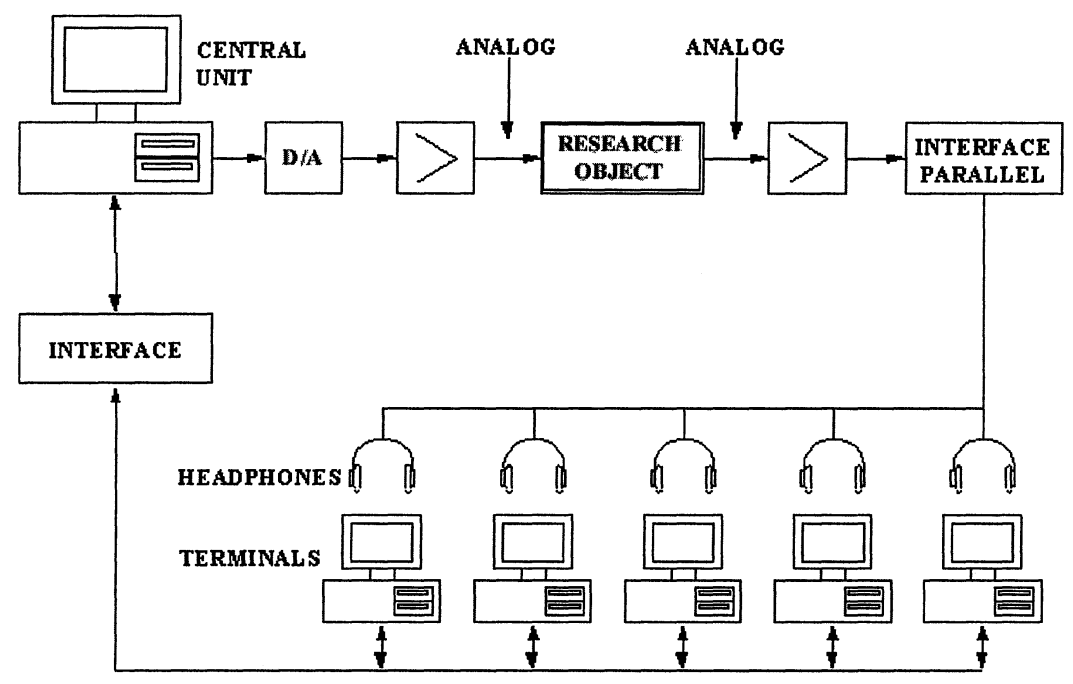

DATA BUS (ANSWERS) SYNCHRO

Figure 1. The measuring system for assessment of logatom intelligibility of telecommunication chains.

The presentation of logatoms is provided as following: a defined number of alternative logatoms chosen from all version of particular logatom, and in the next step, the transmitted (proper) logatom is set into one position, chosen randomly. After the listener's choice of a given logatom, the program compares the transmitted logatom with the chosen one. When the comparison gives the positive result, the value of correct answers is increased. After the measurement process, the result is presented on the screen.

The described measurement system allows also to generate logatoms according to traditional method of intelligibility measurement and it guarantees the same measurement conditions of test exposure for both: traditional and MIT-FC methods. Thus, the comparison between the results obtained by means of these two methods may be carried out.

In contrast to traditional measurements the MIT-FC test satisfies the demands of fast and effective intelligibility measurements and exhibits the following features, that are especially useful under field conditions:

1. It permits for individual measurements with multiple repetitions for different listeners. 
2. The test signal has a stable and repeatable quality under conditions of multiple reproductions.

3. The scores are obtained just after measurements are completed.

4. The software applied enables an easy installation of upgradeable applications as a recalculation of the intelligibility scrores to MOS quality scale, graphical presentation of the results, comparison of subjective scores with objective predictors.

5. The intelligibility measurements may be carried out under conditions of direct binaural reception of test material or via headphones (single or double-ear reception).

The test material, the organization of measurements and the training of the listeners is consistent with the Polish Standard [11, 12].

\section{EXPERIMENT I - ASSESSMENT OF SOFTWARE SOLUTIONS}

In the preliminary experiment a measurement of the quality evaluation of speech transmission over Internet network with usage the most popular application (Netmeeting, ICQPhone, Phonefree, Paltalk, Firetalk Basic) was done. The measurements were done with three described methods.

The subjective quality evaluation of speech transmission over IP networks was done with the logatom intelligibility and ACR methods. The number of listeners depended on used method, i.e. 12 listeners in the ACR method and 6 listeners in the logatom intelligibility method. Listeners for the logatom intelligibility method were chosen according to the project of Polish Standard PN-V-90002 [12] and for the ACR method according to recommendation of ITU-T P.800 [5]. Measurements were done according to adequate recommendations $[5,11,12]$ at Institute of Telecommunication and Acoustics at Wroclaw University of Technology. The testing material consisted of phonetically and structurally balanced logatoms and senteces lists. It was recorded on the digital recorder and after cutting into logatoms and sentences and labeling uploaded to the computer. According to the project of the Polish Standard PN-V-90002 testing signals were sampled at frequency of $16 \mathrm{kHz}$ and 16bit accuracy. The signals were transmitted over the IP network done in hardware and software realizations.

The testing material was presented to listeners. Each measure point (with different conditions of transmission) consisted of three 100-logatom lists (for the logatom intelligibility method) and 10 sentences pronounced fifty-fifty by man and woman (for the ACR method). In the ACR method, listeners assessed the quality of speech transmission in range from 1 to 5 according to the MOS speech quality scale and the $\mathrm{MOS}_{\mathrm{LE}}$ listening-effort scale. 
The MOS index for methods based on measurement of logatom intelligibility was obtained according to the conversion rate given by Polish Standard no PN-90/T-05100 [11] and Figure 2. The quality standards given in the Polish Standard PN-90/T-01500 and characteristics presented on Figure 2 were obtained after following the method of multiple regression for measurement quality methods such as logatom intelligibility, sentence intelligibility, the number of return questions at the reception of sentence messages and the average subjective ratings evaluation $[8,10]$. Obtained results are presented in table 1

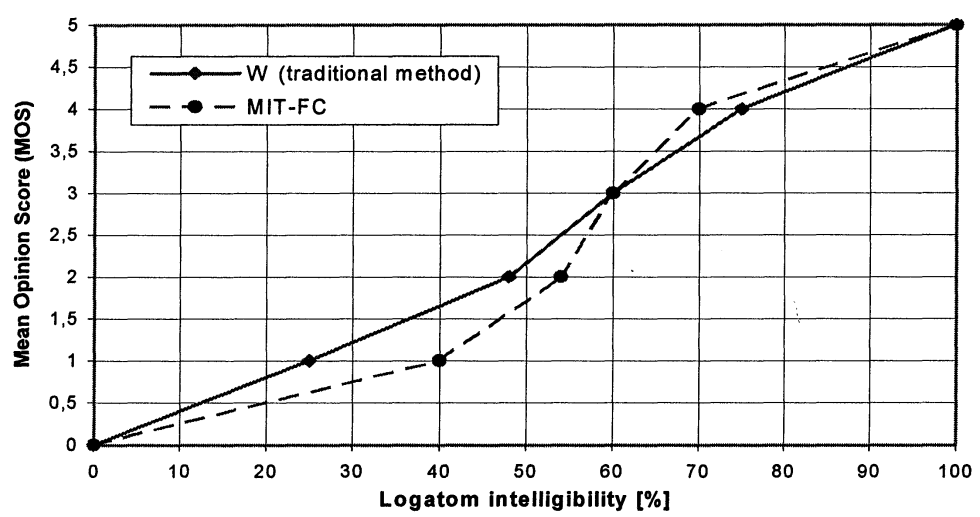

Figure 2. Relationship between MOS and logatom intelligibility measured with traditional and MIT-FC method for analogue telephone chain.

\section{EXPERIMENT II - ASSESSMENT OF THE INFLUENCE OF LOST PACKETS}

Most of the IP telephones users are not able to notice the delays of $100 \mathrm{~ms}$. If the delay is between 100 and $400 \mathrm{~ms}$ (Figure 3 ), the user can notice insignificant delay in the reaction of the second speaker. The delays longer than $400 \mathrm{~ms}$ are clearly noticeable and problems with a natural communication can occur [13].

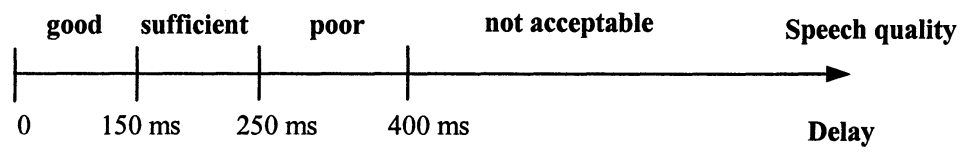

Figure 3. The influence of delay made by the telecommunication channel on the speech quality assessment. 
Table1. Subjective scores of quality of speech transmitted over IP networks

\begin{tabular}{|c|c|c|c|c|c||c|c|c|}
\hline \multirow{2}{*}{ System } & \multicolumn{2}{|c|}{ ACR } & \multicolumn{2}{|c|}{$\mathrm{W}_{\mathrm{L}}$ traditional method } & \multicolumn{3}{|c|}{ MIT-FC } \\
\cline { 2 - 9 } & MOS & $\mathrm{MOS}_{\mathrm{LE}}$ & $\begin{array}{c}\mathrm{W}_{\mathrm{L}} \\
{[\%]}\end{array}$ & QS. & MOS $_{\mathrm{W}}$ & $\begin{array}{c}\mathrm{W}_{\mathrm{L}} \\
{[\%]}\end{array}$ & QS. & MOS $_{\mathrm{M}}$ \\
\hline $\begin{array}{c}\text { Netmeeting CELP } \\
4,8 \text { kbps }\end{array}$ & 3,2 & 3,5 & 72,2 & $\mathrm{II}$ & 3,8 & 79,8 & $\mathrm{I}$ & 4,3 \\
\hline $\begin{array}{c}\text { Netmeeting G.723 } \\
6,8 \text { kbps }\end{array}$ & 3,5 & 3,6 & 79,8 & $\mathrm{I}$ & 4,2 & 85,6 & $\mathrm{I}$ & 4,5 \\
\hline ICQ Phone & 3,6 & 3,7 & 87,2 & $\mathrm{I}$ & 4,5 & 77,0 & $\mathrm{I}$ & 4,2 \\
\hline Phonefree G.723 & 3,4 & 3,9 & 69,3 & $\mathrm{II}$ & 3,6 & 67,7 & $\mathrm{II}$ & 3,9 \\
\hline Paltalk & 3,2 & 3,8 & 76,6 & $\mathrm{I}$ & 4,1 & 82,5 & $\mathrm{I}$ & 4,4 \\
\hline Firetalk Basic & 1,4 & 1,2 & 31,6 & $\mathrm{IV}$ & 1,3 & 47,6 & $\mathrm{IV}$ & 1,5 \\
\hline $\begin{array}{c}\text { Telephon } \\
\text { exchange IP Cisco }\end{array}$ & 3,6 & 3,7 & 79,8 & $\mathrm{I}$ & 4,2 & 82,7 & $\mathrm{I}$ & 4,4 \\
\hline
\end{tabular}

MOS - mean opinion score according to listening-quality scale, $\mathrm{MOS}_{\mathrm{LE}}-$ mean opinion score according to listening-effort scale, $\mathrm{MOS}_{\mathrm{W}}$ - mean opinion score according to logatom intelligibility (traditional method), $\mathrm{MOS}_{\mathrm{M}}$ - mean opinion score according to logatom intelligibility (MIT-FC method), $\mathrm{W}_{\mathrm{L}}$ - logatom intelligibility, QS - quality standard

After the exceed of the acceptable time of delay of the packet, the packet is treated as the lost one. The reaction of the receiving side of the telecommunication channel can be the replacement of the lost packet with:

1. the silence,

2. the preceding or following packet,

3. the signal with the spectrum close to the speech signal spectrum,

4. the interpolation or extrapolation of the signal.

The replacement of the lost packet with the silence interval is an easy option but doesn't give satisfactory effects (disturb the user and the loose of the packet is clearly noticeable).

Another solution is to replace the lost packet with the neighboring packet. Often this kind of lost is not noticed by the user and can be treated as a distortion (one sound is longer). The usage of interpolation and extrapolation algorithms is the most advanced solutions. As a result, in situation when only few packets are lost, only insignificant signal distortions are noticed. Unfortunately the situation when only single packets are lost is very rare one and the most common is when several packets are lost because the network is overloaded for a short time. 
The choice of the compression method, the delay time, time of speech signal in one packet and the method of replacement of the lost packet have a significant influence on the quality of the provision of services.

Until now, the experiences of research made on the assessment of the speech transmission quality over the IP networks have shown that the packet loosing have big influence on the quality of received speech signal. It was decided to make a research of the influence of the length of a packet and the frequency of the appearing of the lost packets on the logatom intelligibility of the transmitted speech signal.

In order to carry out the experiment, there was designed a software simulator of the telecommunication channel, which was inserting in controlled way the distortions in transmitted speech signal. Two parameters were researched: the length of a single packet $T$ (i.e. the time of the speech signal carried in a single packet) and the probability of the packet loss $P(n)$. The simulator didn't take into consideration changes of the delays and the function of probability of the packet loss had an even distribution. The simulator was working on sound files in the RIFF (WAV) format. For the experiment it was assumed that in real IP telephony systems each packet carries equal time of speech signal. The testing material for subjective assessment consist of phonetically and structurally balanced logatom lists. Tests were carried out for the values of $T$ parameter: $25,50,100$ and $150 \mathrm{~ms}$, the probability of packet loss $P(n)$ was took from range of 1 to $50 \%$. For the determining of a value of each measure point one logatom list consisting of 100 logatoms was taken. The assessment was done with traditional logatom intelligibility method. The group of six listeners was chosen from men of proper hearing and average experience in using Polish language. Tests were done in the quite room conditions at ITA of Wroclaw University of Technology, according to Polish standard recommendations PN-90/T-05100 [11] and project PN-V-9002 [12].

The results of carried out experiments are shown in Table 2 and on Figure 4. On the basis of these the MOS grade was evaluated according to connection between logatom intelligibility and five levels scale (Figure 2).

For short time silence intervals of $\mathrm{T}=25 \mathrm{~ms}$ the loss of single packets didn't significantly affect speech intelligibility and distortions are received as additive short clicks. Furthermore, listeners complained about tiredness after tests, bigger than in other subjective assessments made for other telephone channels. Even though intelligibility of recorded signals was very high. The reason of this fact can be in the processing of sounds by ear-snail which is a main organ responsible for the perception of acoustic signals inside human inner-ear. Acoustic signal processing inside the ear-snail can be, in simplification, modeled as a bank of pass-band filters, which is kind of frequency-analyzer. 
Table 2. Logatom intelligibility and the MOS grade obtained for speech signal with silence intervals in function of length of packets and the probability of packet loss

\begin{tabular}{|c|c|c|c|c||c|c||c|c||}
\hline \multirow{2}{*}{$\begin{array}{c}\text { Probability of } \\
\text { packet loss } \\
\mathrm{P}(\mathrm{n})\end{array}$} & \multicolumn{2}{|c|}{$\mathrm{T}=25 \mathrm{~ms}$} & \multicolumn{2}{|c|}{$\mathrm{T}=50 \mathrm{~ms}$} & \multicolumn{2}{|c|}{$\mathrm{T}=100 \mathrm{~ms}$} & \multicolumn{2}{|c|}{$\mathrm{T}=150 \mathrm{~ms}$} \\
\cline { 2 - 8 } & $\mathrm{W}_{\mathrm{L}}$ & $\mathrm{MOS}$ & $\mathrm{W}_{\mathrm{L}}$ & $\mathrm{MOS}$ & $\mathrm{W}_{\mathrm{L}}$ & $\mathrm{MOS}$ & $\mathrm{W}_{\mathrm{L}}$ & $\mathrm{MOS}$ \\
\hline$[\%]$ & {$[\%]$} & & {$[\%]$} & & {$[\%]$} & & {$[\%]$} & \\
\hline 1 & 97,0 & 4,90 & 94,5 & 4,80 & 94,8 & 4,80 & 94,3 & 4,78 \\
\hline 2 & 96,5 & 4,85 & 92,8 & 4,70 & 93,8 & 4,75 & 90,8 & 4,63 \\
\hline 5 & 93,0 & 4,72 & 91,0 & 4,65 & 89,2 & 4,58 & 84,0 & 4,35 \\
\hline 10 & 89,7 & 4,58 & 84,3 & 4,38 & 83,2 & 4,32 & 76,3 & 4,05 \\
\hline 15 & 86,0 & 4,45 & 79,2 & 4,15 & 77,8 & 4,10 & 67,8 & 3,50 \\
\hline 20 & 82,3 & 4,30 & 76,0 & 4,02 & 70,8 & 3,70 & 60,0 & 3,00 \\
\hline 30 & 74,7 & 3,45 & 66,7 & 3,45 & 60,5 & 3,03 & 49,8 & 2,15 \\
\hline 40 & 63,2 & 3,20 & 52,8 & 2,40 & 50,8 & 2,25 & 37,2 & 1,50 \\
\hline 50 & 45,8 & 1,90 & 40,8 & 1,68 & 28,3 & 1,15 & 26,5 & 1,06 \\
\hline
\end{tabular}

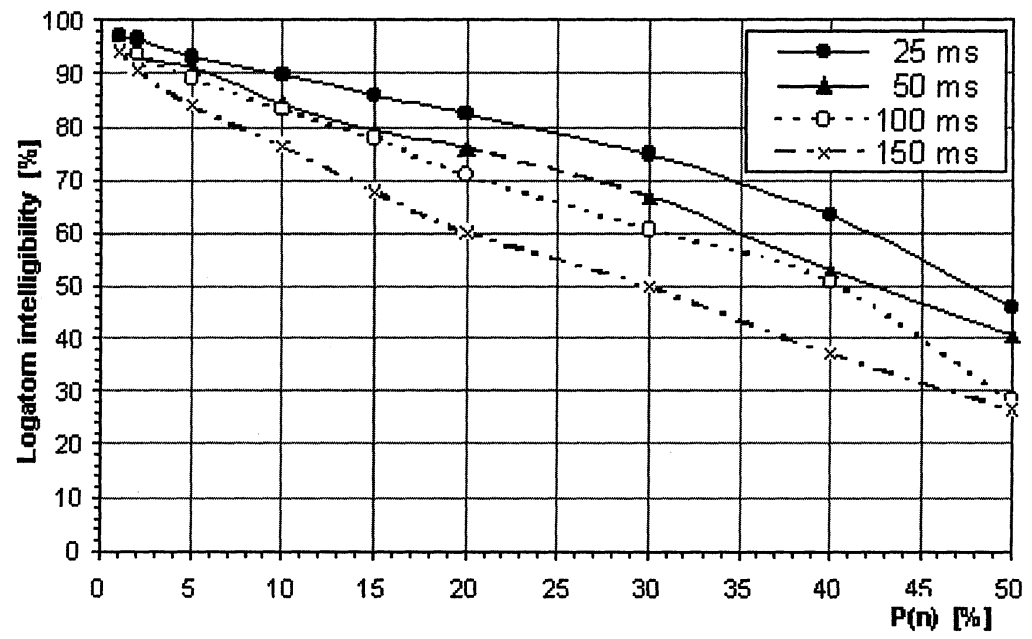

Figure 4. The graph of logatom intelligibility in function the probability of packet loss $W_{L}=f(P(n))$, for time $T \in\{25,50,100,150\} \mathrm{ms}$ 


\section{CONCLUSIONS}

The analysis of the methods of quality evaluation of speech transmission showed that the ACR method, letting obtain speech quality in five levels scale directly, is the most popular nowadays, because it allows fast and not expensive (comparing to other subjective methods) measurement. It makes possible making research for various objects and values of parameters which can influence the quality of transmission and coding.

The results obtained with this method correspond to the opinion expressed by average user. Moreover, the ACR method is standardized in ITU-T P.800 recommendations [5]. The method is not precise in definition of tasks for listeners (different quality scales) and listeners are naturally not able to make stable and objective opinion in point scale of such feature as "quality of speech transmission", which cam be considered as a main disadvantage. Thus, it is necessary to do the measurement using a large group of listeners (larger than 12), more voices (minimum 2 male and 2 female), proceed careful training of listeners and using the reference unit (i.e. MNRU) letting for normalization and averaging of results. On the other hand, subjective methods of measurement of intelligibility have precisely defined tasks for the group of listeners and received results are stable for the same transmission conditions with one disadvantage, which is timeconsuming. The method of modified intelligibility test with alternative choice (MIT-FC), letting for the significant decrease of time-consuming parameter is as fast as the ACR method, allows to determine the quality of speech transmission in five levels scale and the tasks for a group of listeners is better defined.

On the basis of received results of the assessment of the influence of lost packets, it can be noticed that, what was predictible, the intelligibility function is changing monotonous (Figure 4). Surprising was a big convergence of results for cases when $\mathrm{T}=50 \mathrm{~ms}$ and $\mathrm{T}=100 \mathrm{~ms}$. The usage of short time packets $(25 \mathrm{~ms})$ led to significant intelligibility improvement. In the real IP telephony systems it can be, however inconvenient to control such short packets. Obtained results let to count from logatom intelligibility into MOS grate for certain realizations of transmission channels.

Despite of its simplicity, realized simulator of VoIP channel, let to control addition of distortion to transmitted speech signal. Similar solution is the MNRU system (Modulated Noise Reference Unit) recommended by ITU-T. It let to control of addition correlated with transmitted signal noise in order to the simulation of narrow-banded systems coder-decoder of speech signal. Farther research will led to more accurate description of influence of certain parameters characteristic for the packet transmission, in particular VoIP, and for the subjective speech quality and intelligibility assessment. 
Knowing the relations between these parameters can led to realization of fully objective (instrumental) methods of speech quality and intelligibility methods which results would be correlated with subjective ones.

Presented results have only a preliminary character. They are the first step in just started research work into subjective and objective validation of speech transmission in networks using the IP protocols.

\section{REFERENCES}

1. Basciuk K., Brachmanski S., The automation of the subjective measurements of logatom intelligibility, 102-nd Audio Engineering Society Convention, Munich 1997, Preprint 4407 (A5).

2. Brachmanski S., Choosing optimum number of test items in subjective logatom measurements, XLVII Open Seminar on Acoustics OSA'95, Warszawa-Białowieża 1995, pp. 423-428 (in Polish).

3. Brachmanski S., Phonetic structure of test material used in measurements of speech quality of ACR method, XLVIII Open Seminar on Acoustics OSA'2001, Wroclaw 2001, pp. 127-132 (in Polish).

4. Davies D.D., Davies C., Application of speech intelligibility to sound reinforcement, J. Audio Eng. Soc., vol.37, no.12, 1989, pp. 1002-1018.

5. ITU-T Recommendation P.800 (1996), Method for subjective determination of transmission quality.

6. ITU-T Recommendation P.810 (1996), Modulated Noise Reference Unit (MNRU).

7. Mackie K., Assessment of evaluation measures for processed speech, Speech Comm., 6, 1987, pp. 309-316.

8. Majewski W., Basztura Cz., Myślecki W., Relation between speech intelligibility and subjective scale of speech transmission quality, $7^{\text {th }}$ FASE Symposium, Proceedings SPEECH'88, Edinburg, 1988, pp.719-726.

9. Majewski W., Myślecki W., Baściuk K., Brachmański S., Application of modified logatom intelligibility test in telecommunications, audiometry and room acoustics., Proceedings $9^{\text {th }}$ Mediterranean Electrotechnical Conference Melecon'98, Tel-Aviv, Israel, 1998, pp 25-28.

10. Myslecki W., Majewski W., Basztura Cz., Quantitative and qualitative standards of transmission evaluation for Polish, Proc. $9^{\text {th }}$ Conference on Acoustics, Budapest, 1988, pp 126-131.

11. PN-90/T-05100, Analog Communication Systems. Specifications and Methods for Measurement of Logatom Intelligibility, Polish Standard.

12. PN-V-90002, Digital Communication Systems. Specifications and Methods for Measurement of Logatom Intelligibility, Polish Standard.

13. Schulz T., Voice over IP, (white paper), Eicon Technology Corporation, 2000 http://www.eicon.com/disvpri/whtpap4.htm\#conventional telephony. 